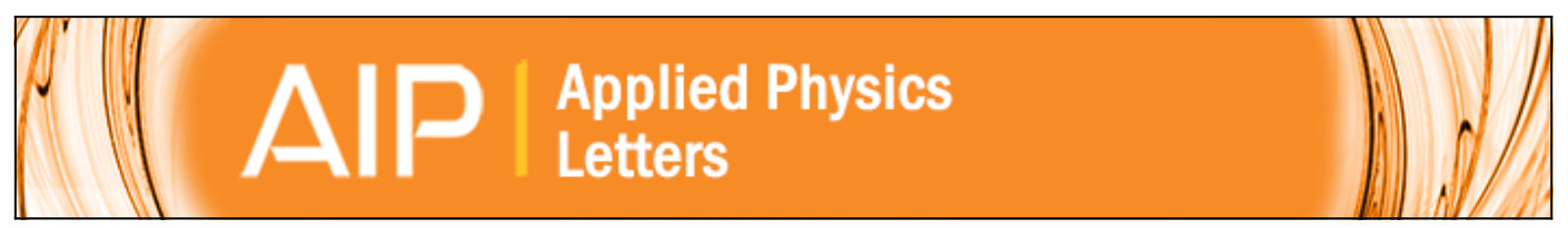

\title{
Asymmetric coalescence of reactively wetting droplets
}

C. X. Zheng, W. X. Tang, and D. E. Jesson

Citation: Applied Physics Letters 100, 071903 (2012); doi: 10.1063/1.3684616

View online: http://dx.doi.org/10.1063/1.3684616

View Table of Contents: http://scitation.aip.org/content/aip/journal/apl/100/7?ver=pdfcov

Published by the AIP Publishing

$A$ AlP

Create a profile.

Sign up today! 


\title{
Asymmetric coalescence of reactively wetting droplets
}

\author{
C. X. Zheng, W. X. Tang, and D. E. Jesson a) \\ School of Physics, Monash University, Victoria 3800, Australia
}

(Received 8 December 2011; accepted 24 January 2012; published online 13 February 2012)

\begin{abstract}
Coalescence of droplets during reactive wetting is investigated for the liquid $\mathrm{Ga} / \mathrm{GaAs}(001)$ system. In situ mirror electron microscopy reveals that coalescence predominantly involves the motion of one reactive droplet relative to the other. This behaviour differs significantly from coalescence in non-reactive systems and is associated with contact line pinning at a ridge/etch pit edge which is identified using atomic force microscopy and selective etching. A simple geometrical model is presented to describe the pinning. (C) 2012 American Institute of Physics. [doi:10.1063/1.3684616]
\end{abstract}

The dynamics of droplet coalescence on surfaces at ambient temperatures is an active area of research. ${ }^{1-3}$ Unlike freely suspended droplets, the contact-line at the liquidsolid-gas interface strongly influences the coalescence kinetics of sessile drops. Analysing droplet kinetics is therefore an important means of studying the contact line motion itself. Studies of coalescence have primarily been restricted to non-reactive droplets. However, there is also significant interest in the contact line dynamics of reactive systems ${ }^{4-8}$ which are of appreciable technological importance. ${ }^{9-11}$ At high temperatures, the spreading of molten metal droplets is accompanied by chemical reactions or interdiffusion at the solid-liquid interface which may deform planar substrates by mass-transport and change the kinetics via ridge formation at the contact-line. Studies of such phenomena have led to important new insights into the kinetics of reactive spreading. ${ }^{4-11}$ However, in comparison with liquids at ambient temperatures,${ }^{1-3}$ droplet coalescence in reactive systems is still poorly understood.

Here, we present a study of droplet coalescence in a model reactive system; liquid Ga on GaAs (001). We identify ridge formation at the contact line by selective etching and atomic force microscopy. The dynamics of reactive droplet coalescence is then studied in real-time by mirror electron microscopy (MEM) $)^{12,13}$ and is found to differ significantly from the behaviour of non-reactive systems, such as low viscosity liquids. A distinctive feature is the motion of one droplet relative to the other. We associate this with enhanced pinning of the contact-line at the ridge/etch pit which subsequently planarises on exposure to vacuum following coalescence.

$\mathrm{Ga}$ droplets were formed on undoped, epi-ready grade GaAs (001) wafers with an actual orientational accuracy of $\pm 0.1^{\circ}$. Wafers were outgassed at $300^{\circ} \mathrm{C}$ under ultra-highvacuum conditions for $24 \mathrm{~h}$ in an Elmitec low energy electron microscope (LEEM) III system. The sample was then flashed up to $600^{\circ} \mathrm{C}$, followed by annealing at $580^{\circ} \mathrm{C}$ for $2 \mathrm{~h}$, to remove the surface oxide. Ga droplets were created by annealing above the planar surface congruent evaporation temperature at $670^{\circ} \mathrm{C}$ and were imaged using MEM. ${ }^{13-16}$ Droplet motion was observed ${ }^{14,15}$ and movies of coalescence events were recorded. The sample was then quenched to

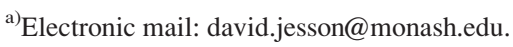

room temperature and droplets were imaged ex situ by atomic force microscopy (AFM) in non-contact mode. To characterise the morphology of the liquid-solid interface, the Ga droplets were selectively etched away using $10 \mathrm{~mol} / \mathrm{L}$ $\mathrm{NaOH}$ solution at $50{ }^{\circ} \mathrm{C}$ which preserved the GaAs surface. The sample was then reimaged by AFM.

At temperatures of $670{ }^{\circ} \mathrm{C}, \mathrm{Ga}$ droplets readily etch $\mathrm{GaAs}^{17-19}$ and also move across the surface driven by a disequilibrium between the droplet and the surface during Langmuir evaporation. ${ }^{14,15}$ Fig. 1(a) contains an AFM image of a droplet which has moved across the slightly rough epi-ready surface leaving behind a smooth trail. A line trace of the droplet and trail is contained in panel (b). Panel (c) contains an AFM image of the etch pit geometry underneath a droplet following selective etching of Ga. The line trace shown in panel (d) clearly identifies a ridge (arrowed) which forms a rim around the etch pit. This appears similar in form to the ridge observed by Somaschini et al. in droplet epitaxy. ${ }^{20}$

Fig. 2(a) displays an AFM image of droplet A that has undergone a coalescence event with a second droplet which had previously occupied the adjacent etch pit B. A line trace across etch pit B and trail as indicated by the white line is contained in (b). Here, there is an absence of a ridge at the edge of the etch pit (cf., panel 1(d)). An AFM image of a post-coalescence geometry similar to panel 2 (a) is shown in panel 2(c) following selective etching of the surface Ga. The line trace corresponding to the horizontal white line is shown in panel (d). Etch pit $\mathrm{C}$ clearly contains a ridge similar to that observed in panel 1(d). We can therefore deduce that, since ridges are associated with the etch pits of isolated droplets (Fig. 1(d)) and are absent from etch pits exposed by droplets which have been displaced by coalescence events (Fig. 2(b)), the droplet which previously occupied etch pit D in Fig. 2(c) was absorbed into a droplet occupying etch pit $\mathrm{C}$ by coalescence.

The absence of ridges at the edges of etch pits exposed by coalescence events suggests two possibilities. First, it is possible that the ridge is dragged by the contact line of the coalescing droplet such that it is erased from the surface. Alternatively, if the droplet coalescence is fast on the time scale of ridge formation/dissolution, the ridge may be left exposed to vacuum and subsequently planarise. To further investigate this issue we turn to MEM which is capable of studying coalescence events of reactive droplets in real-time 

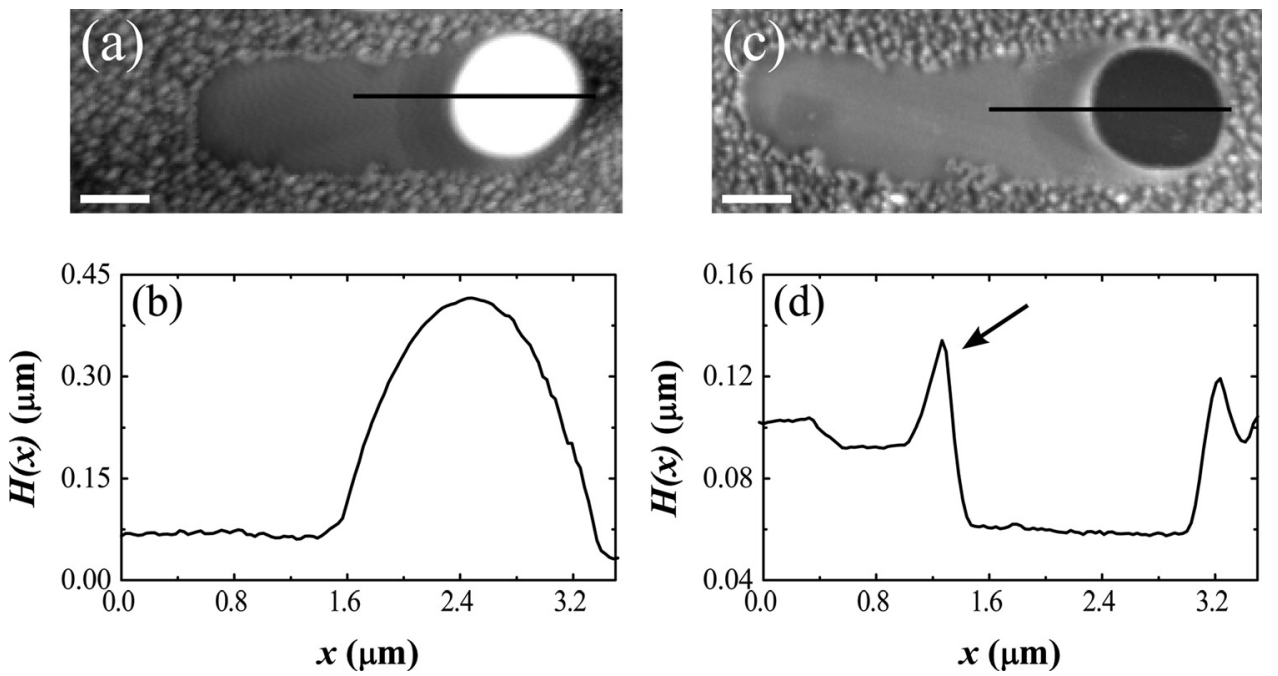

FIG. 1. AFM images of Ga-rich liquid droplets on GaAs (001). (a) A droplet has left behind a smooth trail on the slightly rough epi-ready wafer surface. A line trace corresponding to the line shown is given in (b). (c) AFM image following selective etching of Ga revealing the etch pit geometry beneath a droplet. The line trace corresponding to the horizontal dark line is contained in (d). A ridge (arrowed) forms a rim around the etch pit. Scale markers in (a) and (c) are $1 \mu \mathrm{m}$ across. at elevated temperatures. Images taken from a MEM movie of $\mathrm{Ga}$ droplet coalescence at $670{ }^{\circ} \mathrm{C}$ are contained in Fig. 3. The droplets appear as uniform dark discs somewhat larger than the actual droplet, surrounded by a concentric bright halo. $^{14,15}$ In panel (a), droplet 1 translates across the substrate and coalesces with droplet 2 . The coalescence event is rapid, occurring in less than a movie frame $(0.1 \mathrm{~s})$ giving a minimum contact line velocity of $18 \mu \mathrm{ms}^{-1}$. It is also highly asymmetric in nature involving the slippage and motion of droplet 1 relative to droplet 2 . This exposes the etch pit associated with droplet 1 in panel (b).

To explain the observed coalescence dynamics in reactive systems it is instructive to first consider the coalescence of non-reactive droplets as schematically depicted in Fig. 4(a). Here the droplets are coalescing via a neck region and in Fig. 4(b) we consider the forces acting at contact line posi- tion A. The equilibrium angle of contact $\theta=\theta_{0}$ for an isolated droplet is usually related to surface tensions via Young's equation ${ }^{21}$

$$
\gamma_{v s}-\gamma_{l s}=\gamma_{v l} \cos \theta_{0}
$$

where $\gamma_{v s}, \gamma_{l s}$ and $\gamma_{v l}$ are the respective tensions of the vapour-solid, liquid-solid and vapour-liquid interfaces. This can be simply derived by assuming force equilibrium at the contact line in a direction parallel to the substrate. However, during coalescence the neck region (Fig. 4(a)) will result in a reduction in $\theta$ such that $\theta<\theta_{0}$. This will unbalance the surface tensions creating a net force on the contact line which will move it to the right (ignoring any pinning due to surface roughness). It is therefore usual to observe both droplets moving during coalescence in non-reactive systems. ${ }^{1-3}$
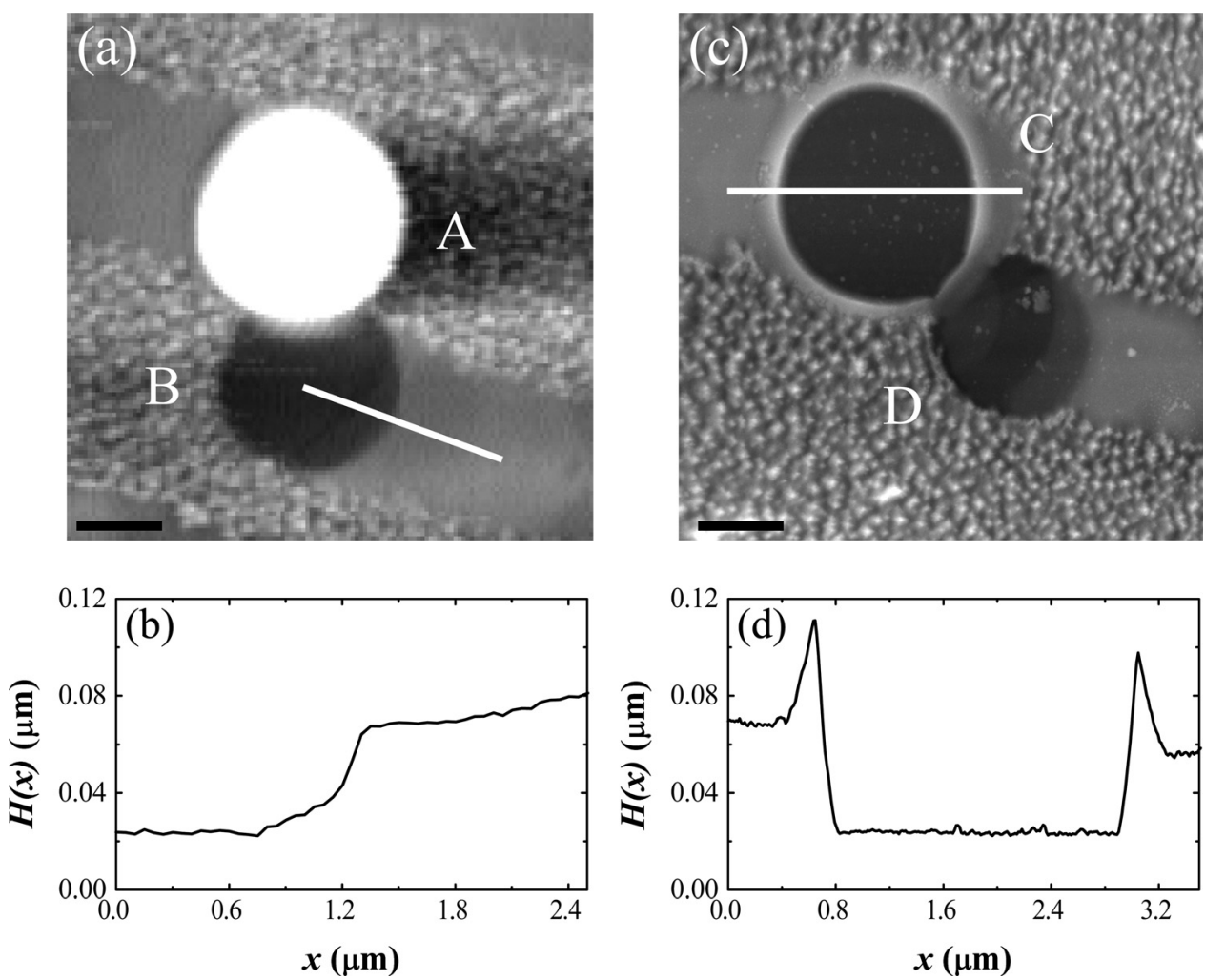

FIG. 2. AFM images of Ga-rich liquid droplet coalescence events on GaAs (001). (a) A droplet previously occupying etch pit B has coalesced with droplet A. (b) Height profile of etch pit B corresponding to the line shown in panel (a). No ridge is present at the edge of the etch pit exposed by coalescence. (c) AFM image of a coalescence event of geometry similar to panel (a) following selective etching of $\mathrm{Ga}$. A line trace of etch pit B is contained in (d) for the line shown in panel (c). Scale markers in (a) and (c) are $1 \mu \mathrm{m}$ across. 

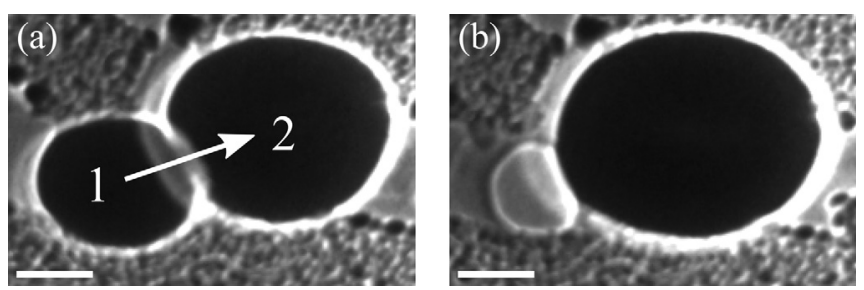

FIG. 3. Images taken from a MEM movie of Ga droplet coalescence at $670^{\circ} \mathrm{C}$. (a) Droplet 1 translates across the surface and coalesces with droplet 2. This occurs in less than $0.1 \mathrm{~s}$ and exposes the etch pit beneath the droplet as shown in (b). The coalescence occurs by the motion of droplet 1 relative to droplet 2. Scale markers in (a) and (b) are $1.5 \mu \mathrm{m}$ across.

The lateral force balance, at the heart of Young's equation, leaves an unbalanced vertical component so that Eq. (1) inherently assumes the substrate is perfectly rigid and nonreactive. For our reactive liquid $\mathrm{Ga} /$ solid GaAs system, it is possible to form a ridge at the contact line by mass transport, as shown in Figs. 1(d) and 2(d). This allows the equilibration of the vertical force as shown in Fig. 4(d ${ }^{8,22-24}$ which, for an isolated droplet, gives rise to the relationship

$$
\frac{\gamma_{v s}}{\sin \theta_{R}}=\frac{\gamma_{v l}}{\sin \varphi}=\frac{\gamma_{l s}}{\sin \delta}
$$

as derived from Neumann's triangle of forces. Here, $\theta=\theta_{R}$, which exactly balances the surface tensions. However, Gibbs $^{25}$ showed that the contact line remains pinned for a range of contact angles given by the inequality

$$
\theta_{0} \leq \theta \leq(\pi-\varphi)+\theta_{0}
$$

which has important implications for coalescence in reactive systems.

In particular, consider the coalescence of two reactive droplets as depicted schematically in Fig. 4(c). The coalescence events in the MEM movies occur in less than $0.1 \mathrm{~s}$ and so we assume that the ridge geometry is preserved during the process. The surface tensions acting at contact line position B are shown in Fig. 4(d). Following formation of the neck region, $\theta$ will be reduced away from $\theta_{R}$ but, provided $\theta \geq \theta_{0}$, Eq. (3) indicates that the contact line will remain pinned. To unpin the droplet at point $\mathrm{B}$, the applied force per unit length of contact line resulting from a reduction in $\theta$ needs to be greater than $f$ where

$$
f=\gamma_{v l}\left(\cos \theta_{0}-\cos \theta_{R}\right) .
$$

The magnitude of the total force to overcome the pinning will increase with droplet radius and so large droplets will tend to absorb smaller droplets during coalescence without shifting their centre of mass. This explains the asymmetric nature of the coalescence dynamics in reactive systems.

In summary, we have studied droplet coalescence events in a model reactive wetting system. Ridge formation at the contact line changes the coalescence dynamics so that when two droplets touch they coalesce with the resulting centre of mass located at the projected centre of the larger of the two droplets. Following exposure to vacuum, the ridge planarises due to As evaporation and surface diffusion of Ga.
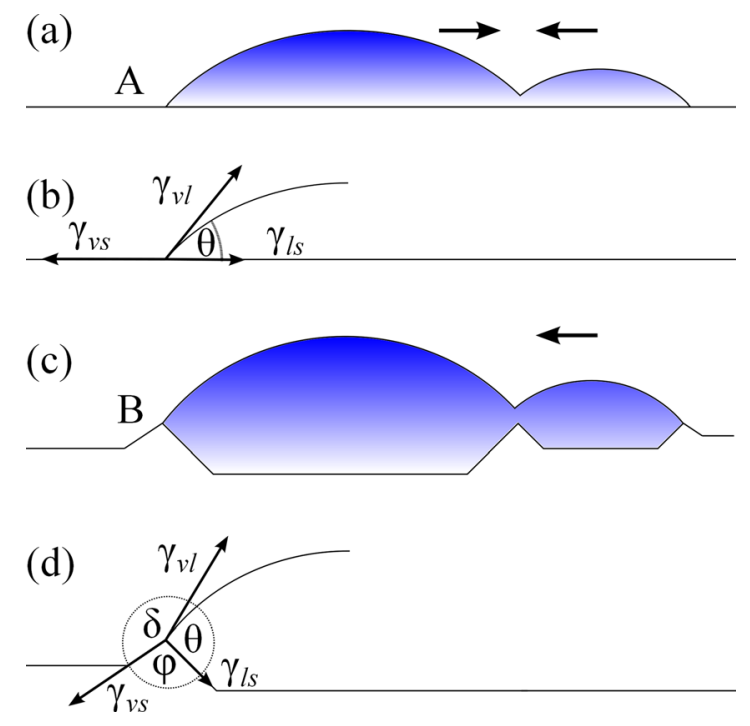

FIG. 4. (Color online) (a) Schematic representation of droplet coalescence in a non-reactive system. The surface tensions acting at contact line position A are shown in (b). (c) Schematic representation of droplet coalescence in a reactive system. The surface tensions acting at contact line position B are shown in (d).

We thank Jerry Tersoff for helpful discussions and Rod Mackie for technical support. D.E.J and W.X.T. acknowledge support by the Australian Research Council (Grant No. DP0985290).

${ }^{1}$ R. Narhe, D. Beysens, and V. S. Nikolayev, Langmuir 20, 1213 (2004).

${ }^{2}$ M. Wu, T. Cubaud, and C.-M. Ho, Phys. Fluids 16, L51 (2004).

${ }^{3}$ W. D. Ristenpart, P. M. McCalla, R. V. Roy, and H. A. Stone, Phys. Rev. Lett. 97, 064501 (2006).

${ }^{4}$ D. Chatain and W. C. Carter, Nat. Mater. 3, 843 (2004).

${ }^{5}$ E. Saiz and A. P. Tomsia, Nat. Mater. 3, 903 (2004).

${ }^{6}$ J. A. Warren, W. J. Boettinger, and A. R. Roosen, Acta Mater. 46, 3247 (1998).

${ }^{7}$ N. Eustathopoulos, Acta Mater. 46, 2319 (1998).

${ }^{8}$ E. Saiz, R. M. Cannon, and A. P. Tomsia, Acta Mater. 48, 4449 (2000).

${ }^{9}$ R. J. Braun, B. T. Murray, W. J. Boettinger, and G. B. McFadden, Phys. Fluids 7, 1797 (1995).

${ }^{10}$ J. C. Ambrose, M. G. Nicholas, and A. M. Stoneham, Acta Metal. Mater. 40, 2483 (1992).

${ }^{11}$ J. C. Ambrose, M. G. Nicholas, and A. M. Stoneham, Acta Metal. Mater. 41, 2395 (1993).

${ }^{12}$ S. A. Nepijko, N. N. Sedov, and G. Schönhense, J. Microsc. 203, 269 (2001).

${ }^{13}$ S. M. Kennedy, C. X. Zheng, W. X. Tang, D. M. Paganin, and D. E. Jesson, Ultramicroscopy 111, 356 (2011).

${ }^{14}$ J. Tersoff, D. E. Jesson, and W. X. Tang, Science 324, 236 (2009).

${ }^{15}$ W. X. Tang, C. X. Zheng, Z. Y. Zhou, D. E. Jesson, and J. Tersoff, IBM J. Res. Dev. 55, 10:1 (2011).

${ }^{16}$ J. Tersoff, D. E. Jesson, and W. X. Tang, Phys. Rev. Lett. 105, 035702 (2010).

${ }^{17}$ C. Chatillon and D. Chatain, J. Cryst. Growth 151, 91 (1995).

${ }^{18}$ M. Zinke-Allmang, L. C. Feldman, and W. van Saarloos, Phys. Rev. Lett. 68, 2358 (1992).

${ }^{19}$ T. D. Lowes and M. Zinke-Allmang, J. Appl. Phys. 73, 4937 (1993).

${ }^{20}$ C. Somaschini, S. Bietti, N. Koguchi, and S. Sanguinetti, Nano Lett. 9, 3419 (2009).

${ }^{21}$ T. Young, Philos. Trans. R. Soc. 95, 65 (1805).

${ }^{22}$ G. R. Lester, J. Colloid Sci. 16, 315 (1961).

${ }^{23}$ Y. Liu and R. German, Acta Mater. 44, 1657 (1996).

${ }^{24}$ M. E. R. Shanahan and A. Carré, Langmuir 11, 1396 (1995).

${ }^{25}$ J. W. Gibbs, The Scientific Papers of J. Willard Gibbs (Dover, New York, 1961), Vol. 1. 